\title{
Frequency of Diabetic Ketoacidosis in Patients with Type 1 Diabetes Using FreeStyle Libre: A Retrospective Chart Review
}

\author{
Ayman A. Al Hayek (D) - Mohamed A. Al Dawish (D)
}

Received: March 23, 2021 / Accepted: April 28, 2021 / Published online: May 19, 2021

(C) The Author(s), under exclusive licence to Springer Healthcare Ltd., part of Springer Nature 2021

\begin{abstract}
Introduction: Diabetic ketoacidosis (DKA) is an acute complication associated with poorly managed or undiagnosed diabetes. DKA is associated with significant morbidity, mortality, and healthcare costs, but can be prevented with appropriate management of diabetes. The FreeStyle Libre is flash glucose monitoring device that measures glucose levels in the interstitial subcutaneous tissue and has been shown to reduce $\mathrm{HbA1c}$, time in hypoglycemia and hyperglycemia, as well as improve health-related quality of life.
\end{abstract}

Methods: A retrospective chart review of patients with type 1 diabetes mellitus (T1DM) and recurrent DKA and who initiated FreeStyle Libre (Abbott Diabetes Care, Alameda, CA, USA) was conducted. DKA frequency and severity, glycated hemoglobin (HbA1c), and frequency of blood glucose monitoring were compared between the 2-year period before FreeStyle Libre initiation and the 2-year period after FreeStyle Libre initiation.

Results: A total of 47 patients with T1DM with recurrent DKA were included. FreeStyle Libre was associated with a reduction in the frequency of DKA events, with a mean of 0.2 (standard deviation [SD] 0.4) events per person during the 2 years after FreeStyle Libre initiation versus 2.9 (SD 0.9) during the 2 years before FreeStyle Libre initiation. Severity of DKA events was also reduced, with fewer severe (before mean 0.3 [SD 0.5] versus after 0.0 [SD 0.0]; $p<0.001)$ DKA events. A reduction in HbA1c (mean 7.4\% [SD 0.5] after versus 9.9\% [SD 1.2] before $[p<0.001])$ and an increase in frequency of blood glucose testing (mean 8.1 scans/day [SD 1.7] after versus 2.2 finger-pricks/day [SD $0.7]$ at before $[p<0.001])$ were also observed.

Conclusion: FreeStyle Libre is associated with a reduction in the frequency and severity of DKA events, reduction in $\mathrm{HbA} 1 \mathrm{c}$, and increase in frequency of blood glucose testing in patients with T1DM and recurrent DKA. The use of such a glucose monitoring tool can help to reduce the burden of morbidity, mortality, and healthcare costs associated with complications of diabetes.

Keywords: Diabetic ketoacidosis; Type 1 diabetes mellitus; Wearable electronic devices

A. A. Al Hayek $(\bowtie) \cdot$ M. A. Al Dawish

Department of Endocrinology and Diabetes,

Diabetes Treatment Center, Prince Sultan Military

Medical City, P.O. Box 7897, Riyadh 11159, Saudi

Arabia

e-mail: ayman.alhayek@yahoo.com 


\section{Key Summary Points}

Why carry out this study?

Acute complications of diabetes, including diabetic ketoacidosis (DKA), pose a significant burden in terms of morbidity, mortality, and healthcare costs, but can be prevented with appropriate management of diabetes.

FreeStyle Libre flash glucose monitoring has been shown to reduce HbA1c and time in hypoglycemia and hyperglycemia, as well as improve health-related quality of time, reduce diabetes distress, and improve sleep quality.

This retrospective chart review aimed to analyze changes in the frequency and severity of DKA events following initiation of FreeStyle Libre.

\section{What was learned from the study?}

FreeStyle Libre is associated with a reduction in the frequency and severity of DKA events.

This glucose monitoring device is a further tool for reducing the recurrence of DKA in addition to interventions such as intensive diabetes management, psychosocial and educational approaches, regular follow-up, and participation of family members in a patient's diabetes management.

\section{DIGITAL FEATURES}

This article is published with digital features, including a summary slide, to facilitate understanding of the article. To view digital features for this article go to https://doi.org/10.6084/ m9.figshare.14495361.

\section{INTRODUCTION}

Type 1 diabetes mellitus (T1DM) is a chronic autoimmune metabolic disorder characterized by a lack of insulin production by beta cells of the pancreatic islets. According to the International Diabetes Federation Diabetes Atlas, published in 2019, approximately 1.1 million individuals aged 19 years and under suffer from T1DM globally [1]. In Saudi Arabia, where rates of T1DM have risen sharply in recent years, 27,800 individuals aged 19 years and under suffer from the condition [2, 3].

Poorly managed or undiagnosed diabetes can result in complications. Such complications include microvascular complications, e.g., retinopathy, neuropathy, and nephropathy, as well as macrovascular complications, e.g., stroke, peripheral arterial disease, and coronary artery disease [4]. Acute complications associated with diabetes include diabetic ketoacidosis (DKA), lactic acidosis, hypoglycemia, and hyperglycemic hyperosmolar state [5]. These acute complications pose a significant burden in terms of morbidity, mortality, and healthcare costs, but can be prevented with appropriate management of diabetes [5].

DKA results from a lack of insulin and increased levels of counter-regulatory hormones such as growth hormone, glucagon, cortisol, and catecholamines, leading to increased production of glucose and ultimately ketonemia and acidosis which can be fatal [6]. DKA is one of the most common causes of death in children with diabetes [7], with cerebral edema being the main underlying cause [8]. In Saudi Arabia, the rate of DKA is high, with between $25 \%$ and $80 \%$ of patients with T1DM developing DKA [9].

Recurrent DKA can lead to an increase in the development of microvascular complications, reduced quality of life, and increased mortality [10]. Among 197 hospital admissions in 92 children with newly diagnosed T1DM aged 8-13 years old, $28 \%(26 / 92)$ of patients experienced three or more hospital admissions, which accounted for $80.2 \%(158 / 197)$ of all of the admissions within this set of children [11]. Factors associated with increased risk of DKA include female gender, young age, early age of 
diabetes onset, lower socioeconomic status, and psychosocial disorders [10]. Risk factors associated with DKA in patients with T1DM in Saudi Arabia include age 13-19 years, female sex, not attending diabetes clinic appointments, and a glycated hemoglobin (HbA1c) of $10 \%$ or higher [12].

New technologies in the field of diabetes, such as continuous glucose monitoring or flash glucose monitoring, enable greater frequency of blood glucose measurement as well as insights into the trends of blood glucose. By improving glycemic control, such technologies are thought to be able to reduce the incidence of complications and improve outcomes in patients with diabetes [13]. FreeStyle Libre (Abbott Diabetes Care, Alameda, CA, USA) is a flash glucose monitoring device that uses a factorycalibrated sensor system to measure glucose levels in the interstitial subcutaneous tissue [14]. It has been demonstrated that the device can provide a number of benefits to the T1DM population, including a reduction in $\mathrm{HbA1c}$ and time in hypoglycemia and hyperglycemia, as well as improved health-related quality of life, reduced diabetes distress, and improved sleep quality [15-18].

This retrospective chart review aimed to analyze changes in the frequency and severity of DKA events following initiation of the FreeStyle Libre flash glucose monitoring system. Further aims were to assess changes in HbA1c and frequency of blood glucose testing following FreeStyle Libre initiation.

\section{METHODS}

\section{Study Design}

This retrospective chart review was conducted in patients with T1DM and recurrent DKA (more than one DKA episode) from the Diabetes Treatment Center, Prince Sultan Military Medical City (PSMMC), Riyadh, Saudi Arabia. All patients with T1DM registered at the Diabetes Treatment Center at PSMMC were considered for inclusion in the study. Patients were included if they were aged 13 years and above, had a diagnosis of T1DM for at least 1 year at FreeStyle
Libre initiation, were first-time users of the FreeStyle Libre system, and had at least one DKA event (excluding the first DKA presentation) at FreeStyle Libre initiation. Patients were also required to have at least 2 years of clinical data after FreeStyle Libre system initiation and full use of FreeStyle Libre for the whole of period following FreeStyle Libre initiation, with evidence of continued dispensing of flash glucose monitoring sensors and documented flash glucose monitoring sensor use for at least $50 \%$ of the time during follow-up. Patients were excluded if they were using other continuous glucose monitoring devices or had used such devices within the 6 months prior to FreeStyle Libre insertion. Patients using insulin pump therapy were also excluded.

All data was collected retrospectively. Baseline data was collected for a period of 2 years, between December 2016 and December 2018, prior to initiation of FreeStyle Libre. Follow-up data was collected for a period of 2 years, between January 2019 and December 2020, following FreeStyle Libre initiation.

Education for self-management is these patients throughout the observation period included regular visits to the diabetes center at PSMMC and regular education on insulin injection and self-monitoring of blood glucose. Education specific to flash glucose monitoring was provided following FreeStyle Libre initiation. The education program [19] was provided through the diabetes educators and one-on-one counseling sessions with the patient's doctor.

\section{Variables}

Demographic (age, sex, weight, and height) and clinical (age at diagnosis, HbA1c, DKA events, severity of DKA events, precipitating factors for DKA events, frequency of blood glucose testing, and total daily insulin dose) data were collected for the 2-year period prior to FreeStyle Libre initiation from electronic medical records. HbA1c, DKA events, severity of DKA events, precipitating factors for DKA events, and frequency of blood glucose testing were similarly abstracted from electronic medical records for the 2-year period following FreeStyle Libre 
initiation. Diabetic ketoacidosis was defined as an episode of hyperglycemia with ketoacidosis, requiring a visit to an emergency department and/or hospital admission. In this study, patients with DKA were those with plasma glucose above $13.8 \mathrm{mmol} / \mathrm{L}$, urinary/serum ketones, serum bicarbonate below $18 \mathrm{mmol} / \mathrm{L}$, and/or arterial/venous $\mathrm{pH}$ less than 7.30. Severity of DKA events was classified as mild, moderate, or severe on the basis of the intensity of the metabolic acidosis and the presence of altered mental status as per the American Diabetes Association grading system [20]. HbA1c (\%) was measured using the COBAS INTEGRA 400 plus/800 analyzers at the central laboratory of PSMMC. During the 2-year period prior to FreeStyle Libre initiation, an average of the three most recent tests performed between December 2016 and December 2018 was used. Similarly, an average of the three most recent HbA1c test results following FreeStyle Libre initiation was used for the period following FreeStyle Libre initiation. Precipitating factors for DKA events were categorized according to the evaluations of the emergency physician or endocrinologist as poor adherence to insulin therapy or insulin omission, diet non-adherence, blood glucose self-monitoring non-compliance, infection, or psychosocial issues. Frequency of blood glucose testing was defined as the mean number of blood glucose tests performed using finger-prick. Frequency of selfmonitoring of blood glucose was documented in the patient's medical records either by the attending emergency room physicians who requested frequency from the patient or by diabetes educators who downloaded blood glucose meter data. Mean number of FreeStyle Libre scans per day was also captured for the period following FreeStyle Libre initiation.

\section{Analysis}

Mean and standard deviation (SD) were calculated for continuous variables and frequencies with proportions for categorical variables. The Q-Q plot and Shapiro-Wilk test were used to assess the distribution of the data. The paired $t$ test (for normally distributed data) or the
Wilcoxon matched-pairs signed-rank test (for non-normally distributed data) was used to assess any differences between the two time periods. Missing data are presented without imputation. StataIC (StataCorp, Version 16) was used for all analyses.

\section{Ethics}

Approval of the study protocol was granted by the Research Ethics Committee of the PSMMC in accordance with the Helsinki Declaration of 1964, revised in 2013 (ethical approval number 1242).

\section{RESULTS}

\section{Cohort Demographics}

Of 1118 patients with T1DM registered at the PSMMC Diabetes Treatment Center, 208 of these had a history of DKA and were considered for inclusion in the study. Fifty-seven of these patients had 2 years of available clinical data on recurrent DKA following initiation of FreeStyle Libre. Ten patients were excluded from the final analysis because of FreeStyle Libre sensor use less than $50 \%$ of the time during the period following FreeStyle Libre initiation $(n=8)$ or use of insulin pump therapy $(n=2)$. A total of 47 patients were included in the analysis (Fig. 1). Patient demographics are shown in Table 1.

\section{Frequency and Severity of Diabetic Ketoacidosis}

A total of 137 DKA events were reported in the 47 patients during the 2 -year period prior to FreeStyle Libre initiation: 73 mild, 49 moderate, and 15 severe. In contrast, only 10 DKA events were reported during the period following FreeStyle Libre initiation in 10 patients: 9 mild and 1 moderate. Frequency of DKA was reduced following FreeStyle Libre initiation, with a mean of 0.2 (SD 0.4) compared with 2.9 (SD 0.9) during the period prior to FreeStyle Libre initiation. Severity of DKA events was also reduced, 


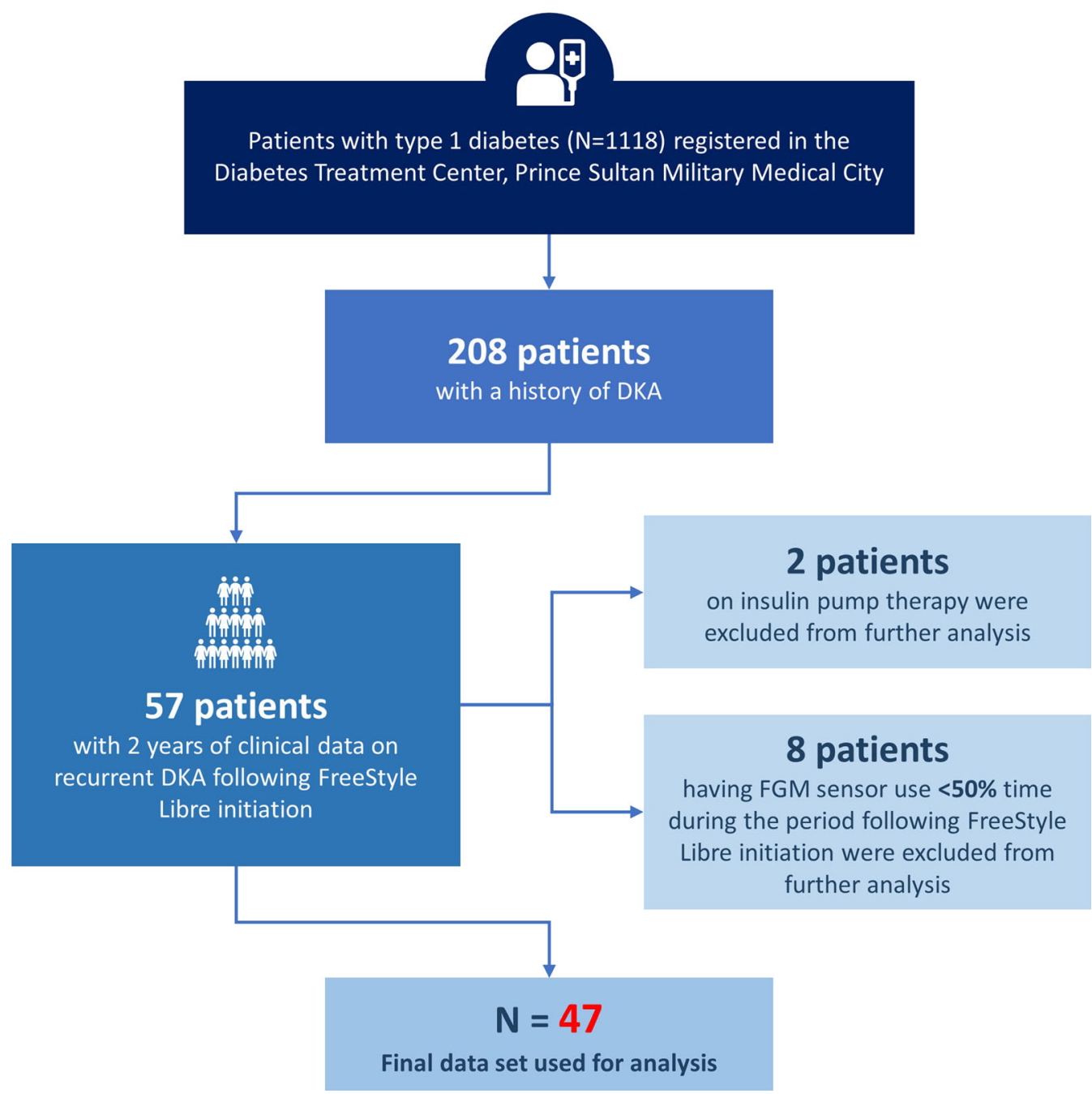

Fig. 1 Study recruitment flow diagram. DKA diabetic ketoacidosis, FGM flash glucose monitoring, RDKA recurrent diabetic ketoacidosis

with fewer severe (prior mean 0.3 [SD 0.5 ] versus after mean 0.0 [SD 0.0]; $p<0.001$ ), moderate (prior mean $1.0[\mathrm{SD} 0.6]$ versus after mean 0.02 [SD 0.1]; $p<0.001$ ), and mild (prior mean 2.9 [SD 0.9] versus after mean 0.2 [SD 0.4$]$; $p<0.001$ ) DKA events (Figs. 2 and 3).

\section{HbA1c}

HbA1c was significantly reduced following FreeStyle Libre initiation, with a mean of $7.4 \%$ (SD 0.5) versus 9.9\% (SD 1.2) during the period prior to FreeStyle Libre initiation $(p<0.001)$ (Table 2).

\section{Frequency of Glucose Testing}

Frequency of glucose testing using finger-prick was significantly decreased in the period following FreeStyle Libre initiation, with a mean of 1.0 finger-pricks per day (SD 0.3) versus 2.2 finger-pricks per day (SD 0.7) during the period prior to FreeStyle Libre initiation $(p<0.001)$. On the other hand, a mean of 8.1 scans per day (SD 1.7) following FreeStyle Libre initiation $(p<0.001$ versus glucose testing using fingerprick prior to FreeStyle Libre initiation) was observed. 
Table 1 Patient demographics

\begin{tabular}{lc}
\hline & $N=47$ \\
\hline Age, mean (SD) & $19.8(6.2)$ \\
Age at diagnosis, mean (SD) & $6.4(3.3)$ \\
Gender, $n$ (\%) & $22(46.8)$ \\
Male & $25(53.2)$ \\
Female & $24.4(2.2)$ \\
BMI kg/m ${ }^{2}$, mean (SD) & $1.3(0.4)$ \\
Total daily insulin dose U/kg, mean (SD)
\end{tabular}

$B M I$ body mass index, $S D$ standard deviation

\section{Precipitating Factors for DKA}

The precipitating factors associated with DKA events in each patient are shown in Table 3. Prior to FreeStyle Libre use, infection (85.1\%), blood glucose monitoring non-compliance (78.7\%), and poor adherence to insulin therapy or insulin omission $(68.1 \%)$ were common precipitating factors for DKA. Poor adherence to insulin therapy or insulin omission (50\%) and infection $(50 \%)$ were the only precipitating factors associated with DKA events following FreeStyle Libre use.

\section{DISCUSSION}

FreeStyle Libre is associated with a reduction in the frequency and severity of DKA events, reduction in $\mathrm{HbA} 1 \mathrm{c}$, and increase in frequency of glucose testing in this population of patients with T1DM with recurrent DKA.

To our knowledge this is one of the first studies of its kind to assess the effect of a flash glucose monitoring device on the frequency and severity of DKA. Our data suggest that FreeStyle Libre is associated with a reduction in the number as well as the severity of DKA events. Results from the RELIEF retrospective database study conducted in France and presented at the American Diabetes Association 80th virtual conference highlighted the capability of FreeStyle Libre to reduce DKA events by $52 \%$ in patients with T1DM [21]. A registry study published by Tauschmann et al. demonstrated that continuous glucose monitoring in children with T1DM reduced the frequency of DKA episodes from $1 \%$ at baseline to $0.5 \%$ at 6 months $(p=0.0055)$ and $0.5 \%$ at 12 months $(p=0.0143)$, with a reduction in event rates from 2.0 events per 100 person-years (95\% CI $1.4-2.9)$ to 1.1 events per 100 person-years $(95 \%$ CI 0.7-1.7) at 12 months $(p=0.0254)$ [22].

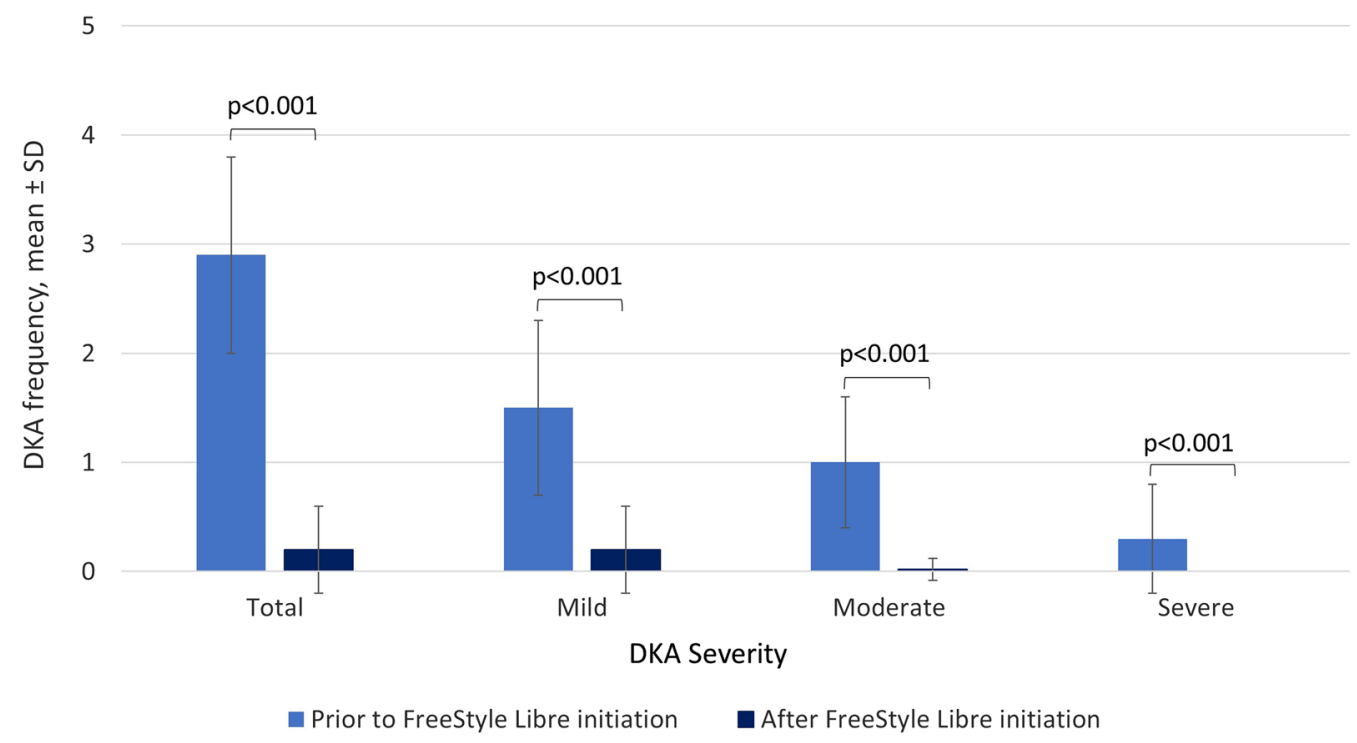

Fig. 2 Mean frequency of DKA events, total and stratified by severity. $D K A$ diabetic ketoacidosis, $S D$ standard deviation 

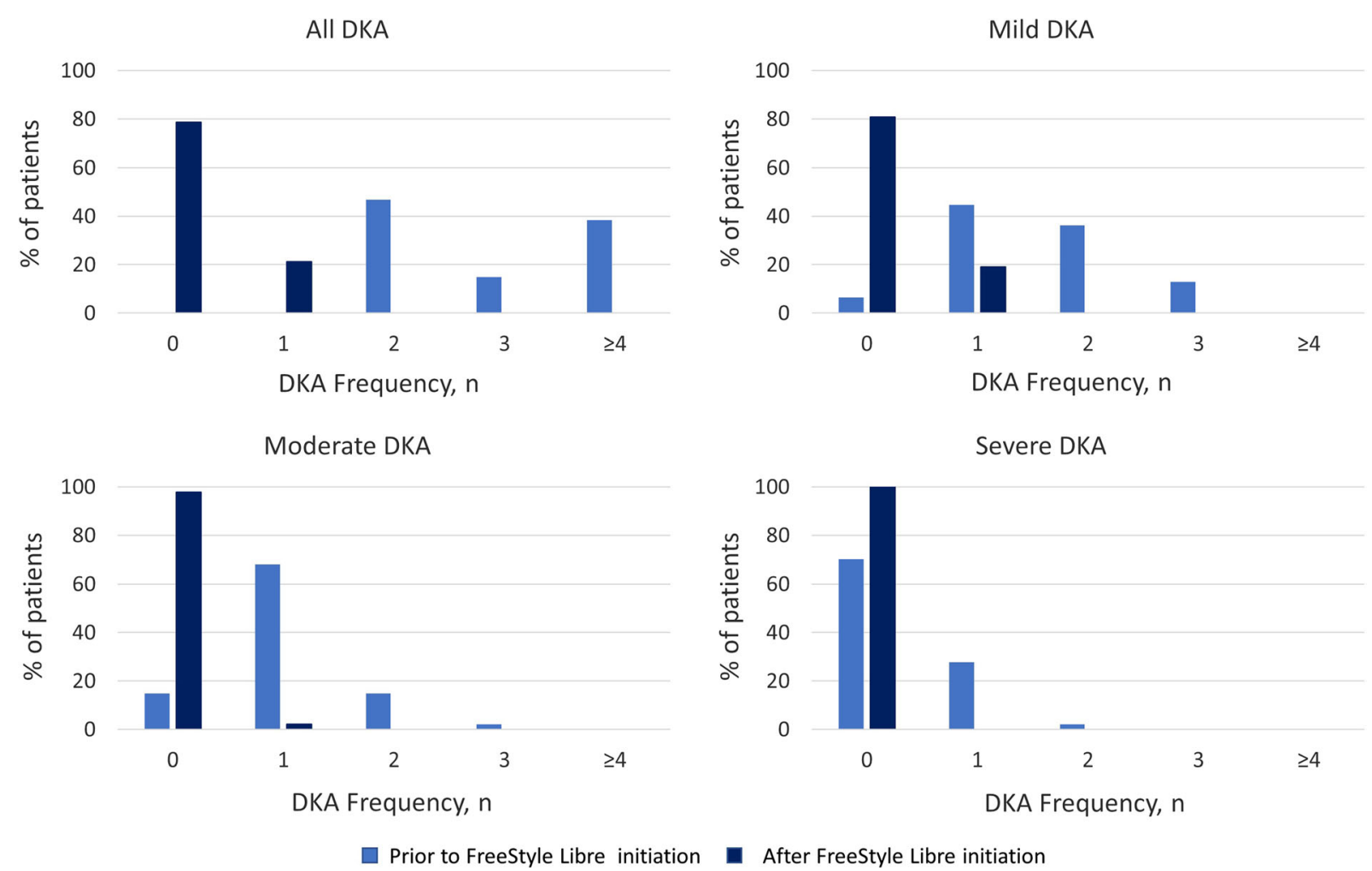

Fig. 3 Frequency of DKA events, total and stratified by severity. DKA diabetic ketoacidosis

Studies have previously reported the most common precipitating factors associated with DKA events. A retrospective cohort study in a tertiary care teaching hospital in Israel demonstrated that issues with insulin therapy, including missed injections, changes in dose or regimen, or pump issues $(40.4 \%)$, and infection $(32.7 \%)$ were the two most common precipitating factors for DKA [23]. Similarly, in a retrospective study conducted in Brazil, medication and diet non-adherence (49\%) and infection (32\%) were the most common precipitating factors for DKA in patients with T1DM [24]. Case reports have recently demonstrated COVID-19 infection to be a precipitating factor for DKA [25]. Our previous study, conducted between May and July 2020, indicated that DKA was the most common reason for hospital admission among patients with T1DM and COVID-19, with a total of five (71.4\%) DKA cases admitted to the hospital for DKA management [26]. In the COVID-19 era, it is important to ensure adequate glycemic control in patients with diabetes, via devices such as FreeStyle Libre, in order to reduce the risk of DKA events.

This study demonstrated a reduction in HbA1c following FreeStyle Libre use. A number of studies have previously demonstrated improvements in HbA1c following the use of flash glucose monitoring. Two meta-analyses were conducted to assess all available evidence from clinical trials and observational studies. Evans et al. included 21 studies that reported changes in HbA1c in 1470 children and adults with T1DM or type 2 diabetes mellitus (T2DM) following initiation of FreeStyle Libre. The meta-analysis demonstrated a reduction in HbA1c of $0.55 \%(95 \% \mathrm{CI}-0.70$ to -0.39$)$ at 2-4 months following initiation of FreeStyle Libre, with no significant difference in change in HbA1c observed between patients with T1DM and patients with T2DM (95\% CI -0.51 to $0.17, p=0.2883$ ) [27]. Gordon et al. evaluated HbA1c data from 34 studies with a total of 5466 patients with T1DM and demonstrated a 
Table $2 \mathrm{HbAlc}$ and frequency of blood glucose testing at baseline and following FreeStyle Libre initiation

\begin{tabular}{llll}
\hline & $\begin{array}{l}\text { Prior to FreeStyle Libre } \\
\text { initiation }\end{array}$ & $\begin{array}{l}\text { After FreeStyle Libre } \\
\text { initiation }\end{array}$ & p value \\
\hline HbAlc, $n(\%)$ & $0(0.0)$ & $9(19.2)$ & $<0.001$ \\
$\leq 7 \%(\leq 53 \mathrm{mmol} / \mathrm{mol})$ & $12(25.5)$ & $38(80.8)$ & \\
$>7 \%$ and $\leq 9 \%(>53$ & $35(74.5)$ & $0(0.0)$ & $<0.001$ \\
and $\leq 75 \mathrm{mmol} / \mathrm{mol})$ & $9.9(1.2)$ & $7.4(0.5)$ & \\
$>9 \%(>75 \mathrm{mmol} / \mathrm{mol})$ & $4(9.5)$ & $23(48.9)$ & \\
Mean $(\mathrm{SD})$ & $38(90.5)$ & $24(51.1)$ & \\
Frequency of blood glucose testing using finger-prick per day, $n(\%)$ & $0(0.0)$ & \\
$\leq 1$ & $0(0.0)$ & 0 & \\
$2-4$ & 5 & $1.0(0.3)$ & \\
$\geq 5$ & $2.2(0.7)$ & $0.001^{*}$ \\
Missing & - & $0(0.0)$ & \\
Mean $(\mathrm{SD})$ & - & $47(100.0)$ & \\
Frequency of FreeStyle Libre scanning per day, $n(\%)$ & $0.1(1.7)$ & \\
$\leq 1$ & - & & \\
$2-4$ & - & & \\
$\geq 5$ & - & & \\
Missing & - & & \\
Mean $(\mathrm{SD})$ & & & \\
\hline
\end{tabular}

HbAlc glycated hemoglobin, $S D$ standard deviation

${ }^{*}$ vs frequency of blood glucose testing using finger-prick

reduction in $\mathrm{HbA} 1 \mathrm{c}$ of $0.41 \%$ ([95\% CI -0.51 to $-0.31] ; p<0.001 ;-4.5$ [95\% CI -5.6 to - 3.3] $\mathrm{mmol} / \mathrm{mol}$ ) following initiation of FreeStyle Libre [28]. Both meta-analyses demonstrated a large heterogeneity in the data, likely due to differences in populations, including variability in baseline HbA1c. Nevertheless, both studies concluded that there is evidence of a reduction in HbA1c, consistent with reduced hyperglycemia [27, 28].

Our data suggest that FreeStyle Libre is associated with an increase in frequency of blood glucose testing, with less finger-prick testing but greater FreeStyle Libre scanning frequency. The association between use of FreeStyle Libre and frequency of glucose testing has also been evaluated in previous studies. A randomized control study involving 64 patients with T1DM aged 13-20 years who were randomly assigned to FreeStyle Libre $(n=33)$ or control $(n=31)$ demonstrated a greater frequency of glucose monitoring in those using FreeStyle Libre at 6 months compared to control, with a difference between groups in the adjusted change at 6 months compared to baseline of 2.8 (95\% CI 1.72-4.65; $p<0.001)$. Whilst FreeStyle Libre users reported an increase in interstitial and capillary checks per day from $1.8 \pm 1.6$ at baseline to $3.8 \pm 3.1$ at 6 months, the control group reported a decrease from $1.9 \pm 3.6$ at baseline to $1.4 \pm 3.0$ at 6 months [29]. A previous study conducted in Saudi 
Table 3 Precipitating factors for DKA events

\begin{tabular}{lll}
\hline $\begin{array}{l}\text { Precipitating } \\
\text { factors, } \boldsymbol{n}(\%)\end{array}$ & $\begin{array}{l}\text { Prior to } \\
\text { FreeStyle Libre } \\
\text { initiation } \\
(\boldsymbol{N}=\mathbf{4 7})\end{array}$ & $\begin{array}{l}\text { After FreeStyle } \\
\text { Libre initiation } \\
(\boldsymbol{N}=\mathbf{1 0})\end{array}$ \\
\hline $\begin{array}{l}\text { Poor adherence to } \\
\text { insulin therapy or } \\
\text { insulin omission }\end{array}$ & $32(68.1)$ & $5(50.0)$ \\
$\begin{array}{l}\text { Diet non-adherence } \\
\begin{array}{l}\text { Blood glucose self- } \\
\text { monitoring non- } \\
\text { compliance }\end{array}\end{array}$ & $17(2.1)$ & $0(0.0)$ \\
$\begin{array}{l}\text { Infection } \\
\text { Psychosocial issues }\end{array}$ & $4(8.5)$ & $0(0.0)$ \\
\hline
\end{tabular}

$D K A$ diabetic ketoacidosis

Arabia also demonstrated an increase in mean frequency of blood glucose testing following 3 months of FreeStyle Libre use in 95 patients with T1DM, from 2.5 times per day (SD 0.9) at baseline to 5.2 times per day (SD 1.2) at 3 months $(p<0.001)$ [16].

Therefore, the use of FreeStyle Libre is a further tool for reducing the recurrence of DKA in addition to interventions such as intensive diabetes management [10], psychosocial and educational approaches [30], regular follow-up, and participation of family members in a patient's diabetes management [31].

Whilst retrospective studies have a number of advantages, such as ease of conducting and no loss to follow-up, limitations include the possibility of selection bias. As all patients registered at the PSMMC were considered for inclusion in the study, those included are likely representative of the population of patients at that given hospital. A further limitation to this study includes the lack of a control group. This was not possible in this study as the clinical practice at PSMMC was to start all patients presenting with DKA on the FreeStyle Libre device; therefore, no controls were available for this study. Furthermore, the comparison of the number of finger-pricks and scanning frequency of FreeStyle Libre as measures of frequency of blood glucose monitoring is not ideal. Nevertheless, FreeStyle Libre scanning is likely to be indicative of glucose monitoring by the user. The small number of patients included in this study is also a potential limitation; however, as a result of the length of follow-up required to measure DKA events, it was not possible to include any additional patients from PSMMC. Future studies might aim to evaluate other complications of diabetes or further confirm the association of FreeStyle Libre use with frequency and severity of DKA events in other hospitals across the country.

\section{CONCLUSIONS}

This study demonstrates that FreeStyle Libre is associated with a reduction in the frequency and severity of DKA events, reduction in HbA1c, and increase in frequency of glucose testing in patients with T1DM and recurrent DKA in Saudi Arabia. The data contribute additional findings pertaining to the advantages of flash glucose monitoring technology in diabetes. Reducing the frequency and severity of DKA events will help to reduce associated morbidity, mortality, and healthcare costs.

\section{ACKNOWLEDGEMENTS}

Funding. No funding or sponsorship was received for this study. The Rapid Service Fee was funded by Abbott Diabetes Care.

Medical Writing Assistance. Medical writing assistance in the preparation of this article was provided by Dr. Abigail Holland of Connect Communications. Support for this assistance was funded by Abbott Diabetes Care.

Authorship. All named authors meet the International Committee of Medical Journal Editors (ICMJE) criteria for authorship for this article, take responsibility for the integrity of the work as a whole, and have given their approval for this version to be published. 
Authorship Contributions. All authors contributed to the study conception and design, data collection, data analysis, drafting and review of the manuscript as well as approval of the final manuscript.

Disclosures. Ayman A. Al Hayek and Mohamed A. Al Dawish report no conflicts of interest.

Compliance with Ethics Guidelines. Approval of the study protocol was granted by the Research Ethics Committee of the PSMMC in accordance with the Helsinki Declaration of 1964, revised in 2013 (ethical approval number 1242).

Data Availability. The datasets generated during and/or analyzed during the current study are available from the corresponding author on reasonable request.

\section{REFERENCES}

1. Patterson CC, Karuranga S, Salpea P, et al. Worldwide estimates of incidence, prevalence and mortality of type 1 diabetes in children and adolescents: results from the International Diabetes Federation Diabetes Atlas, 9th edition. Diabetes Res Clin Pract. 2019;157:107842.

2. International Diabetes Federation. Saudi Arabia Country report 2010-2045; 2019. https:// diabetesatlas.org/data/en/country/174/sa.html. Accessed 2 Mar 2021.

3. Robert AA, Al-Dawish A, Mujammami M, Dawish MAA. Type 1 diabetes mellitus in Saudi Arabia: a soaring epidemic. Int J Pediatr. 2018;2018:9408370.

4. Fowler M. Microvascular and macrovascular complications of diabetes. Clin Diabetes. 2008;26(2): 77-82.

5. Harding JL, Pavkov ME, Magliano DJ, Shaw JE, Gregg EW. Global trends in diabetes complications: a review of current evidence. Diabetologia. 2019;62(1):3-16.

6. Wolfsdorf J, Craig ME, Daneman D, et al. Diabetic ketoacidosis. Pediatr Diabetes. 2007;8(1):28-43.

7. Daneman D. Diabetes-related mortality. A pediatrician's view. Diabetes Care. 2001;24(5):801-2.
8. Wolfsdorf JI, Allgrove J, Craig ME, et al. Diabetic ketoacidosis and hyperglycemic hyperosmolar state. Pediatr Diabetes. 2014;15(Suppl 20):154-79.

9. Zayed H. Epidemiology of diabetic ketoacidosis in Arab patients with type 1 diabetes: a systematic review. Int J Clin Pract. 2016;70(3):186-95.

10. Skinner TC. Recurrent diabetic ketoacidosis: causes, prevention and management. Horm Res. 2002;57(Suppl 1):78-80.

11. Kovacs M, Charron-Prochownik D, Obrosky DS. A longitudinal study of biomedical and psychosocial predictors of multiple hospitalizations among young people with insulin-dependent diabetes mellitus. Diabet Med. 1995;12(2):142-8.

12. Al Hayek AA, Robert AA, Al-Shaikh R, et al. Factors associated with the presence of diabetic ketoacidosis: a retrospective analysis of patients with type 1 diabetes in Saudi Arabia. Diabetes Metab Syndr. 2020;14(6):2117-22.

13. Prahalad $\mathrm{P}$, Tanenbaum $\mathrm{M}$, Hood $\mathrm{K}$, Maahs DM. Diabetes technology: improving care, improving patient-reported outcomes and preventing complications in young people with type 1 diabetes. Diabet Med. 2018;35(4):419-29.

14. Hoss U, Budiman ES. Factory-calibrated continuous glucose sensors: the science behind the technology. Diabetes Technol Ther. 2017;19(S2):S44-50.

15. Dover AR, Stimson RH, Zammitt NN, Gibb FW. Flash glucose monitoring improves outcomes in a type 1 diabetes clinic. J Diabetes Sci Technol. 2017;11(2):442-3.

16. Al Hayek AA, Al Dawish MA. Assessing diabetes distress and sleep quality in young adults with type 1 diabetes using FreeStyle Libre: a prospective cohort study. Diabetes Ther. 2020;11(7):1551-62.

17. Al Hayek AA, Robert AA, Al Dawish MA. Evaluation of FreeStyle Libre flash glucose monitoring system on glycemic control, health-related quality of life, and fear of hypoglycemia in patients with type 1 diabetes. Clin Med Insights Endocrinol Diabetes. 2017;10:1179551417746957.

18. Dunn TC, Xu Y, Hayter G, Ajjan RA. Real-world flash glucose monitoring patterns and associations between self-monitoring frequency and glycaemic measures: a European analysis of over 60 million glucose tests. Diabetes Res Clin Pract. 2018;137: 37-46.

19. Al Hayek AA, Robert AA, Al Dawish MA, Zamzami MM, Sam AE, Alzaid AA. Impact of an education program on patient anxiety, depression, glycemic control, and adherence to self-care and medication 
in type 2 diabetes. J Family Community Med. 2013;20(2):77-82.

20. Kitabchi AE, Umpierrez GE, Murphy $\mathrm{MB}$, et al. Management of hyperglycemic crises in patients with diabetes. Diabetes Care. 2001;24(1):131-53.

21. Idris IADA. FreeStyle Libre system initiation provides dramatic drop in diabetic ketoacidosis rates. Diabetes Obes Metab. 2020;2020:1.

22. Tauschmann M, Hermann JM, Freiberg C, et al. Reduction in diabetic ketoacidosis and severe hypoglycemia in pediatric type 1 diabetes during the first year of continuous glucose monitoring: a multicenter analysis of 3553 subjects from the DPV Registry. Diabetes Care. 2020;43(3):e40-2.

23. Barski L, Nevzorov R, Rabaev E, et al. Diabetic ketoacidosis: clinical characteristics, precipitating factors and outcomes of care. Isr Med Assoc J. 2012;14(5):299-303.

24. Weinert LS, Scheffel RS, Severo MD, et al. Precipitating factors of diabetic ketoacidosis at a public hospital in a middle-income country. Diabetes Res Clin Pract. 2012;96(1):29-34.

25. Reddy PK, Kuchay MS, Mehta Y, Mishra SK. Diabetic ketoacidosis precipitated by COVID-19: A report of two cases and review of literature. Diabetes Metab Syndr. 2020;14(5):1459-62.
26. Al Hayek AA, Robert AA, Alotaibi ZK, Al DM. Clinical characteristics of hospitalized and home isolated COVID-19 patients with type 1 diabetes. Diabetes Metab Syndr. 2020;14(6):1841-5.

27. Evans M, Welsh Z, Ells S, Seibold A. The impact of flash glucose monitoring on glycaemic control as measured by HbA1c: a meta-analysis of clinical trials and real-world observational studies. Diabetes Ther. 2020;11(1):83-95.

28. Gordon I, Rutherford C, Makarounas-Kirchmann K, Kirchmann M. Meta-analysis of average change in laboratory-measured HbA1c among people with type 1 diabetes mellitus using the 14 day flash glucose monitoring system. Diabetes Res Clin Pract. 2020;164:108158.

29. Boucher SE, Gray AR, Wiltshire EJ, et al. Effect of 6 months of flash glucose monitoring in youth with type 1 diabetes and high-risk glycemic control: a randomized controlled trial. Diabetes Care. 2020;43(10):2388-95.

30. Hampson SE, Skinner TC, Hart J, et al. Effects of educational and psychosocial interventions for adolescents with diabetes mellitus: a systematic review. Health Technol Assess. 2001;5(10):1-79.

31. Brandstaetter E, Bartal C, Sagy I, Jotkowitz A, Barski L. Recurrent diabetic ketoacidosis. Arch Endocrinol Metab. 2019;63(5):531-5. 